\title{
Uptake of amino acids by the parasitic, flagellated protist Trichomonas vaginalis
}

\author{
Xun Zuo, † Barbara C. Lockwood $\ddagger$ and Graham H. Coombs
}

Laboratory for Biochemical

Parasitology, Institute of Biomedical and Life Sciences, Joseph Black Building, University of Glasgow, Glasgow G12 8QQ, UK

\author{
Author for correspondence: Graham H. Coombs. Tel: +44 1413304777 . Fax: +44 1413078016. \\ e-mail: graham.coombs@udcf.gla.ac.uk
}

\begin{abstract}
HPLC techniques have been applied to study amino acid uptake and release by Trichomonas vaginalis under a variety of conditions. Studies on the growth of T. vaginalis in complex media and the survival of the parasite in simple media, with and without amino acids and/or maltose, have shown that the growth or survival of $T$. vaginalis is better in the presence of maltose than when it is absent, and that greater amounts of amino acids are consumed by $\boldsymbol{T}$. vaginalis in the absence of maltose. The results are consistent with several amino acids, notably arginine, threonine, leucine and methionine, being used by $T$. vaginalis as energy substrates. $T$. vaginalis released alanine and glycine into the culture media, the excretion being greater in the presence of maltose. These studies have provided new data on the uptake and release of amino acids by $T$. vaginalis and pave the way for detailed analysis of key enzymes and the regulation of the pathways involved.
\end{abstract}

Keywords: Trichomonas vaginalis, amino acid, HPLC

\section{INTRODUCTION}

Trichomonas vaginalis is an anaerobic, flagellated protozoon which causes vaginal trichomoniasis, a major sexually transmitted disease of humans (Honigberg, 1989). The parasite relies heavily on glycolysis for energy production (Müller, 1988, 1991; Coombs \& Müller, 1995), but it has been suggested that arginine may also serve as an energy substrate (Linstead \& Cranshaw, 1983). Exogenous methionine is rapidly taken up and catabolized by the parasite (Thong et al., 1987) and some released products are suggestive of other amino acids also being catabolized (Lockwood \& Coombs, 1991b; North \& Lockwood, 1995). However, there have been no reports on the uptake and utilization of other amino acids by $T$. vaginalis or the extent to which amino acids can support parasite growth and survival in the absence of exogenous carbohydrates. The aim of this study was to determine which amino acids will support the growth or survival of $T$. vaginalis and to analyse the amino acid changes that occur during the incubation of the parasite in different media. The results provide detailed information on the amino acid uptake and release by $T$. vaginalis and so provide important baseline data for further studies on the functional

†Present address: Department of Zoology, College of Biological Science, University of Guelph, Guelph, Ontario, Canada N1G 2W1.

‡Died 8 October 1993. significance and routes of amino acid catabolism by $T$. vaginalis.

\section{METHODS}

Parasite. Trichomonas vaginalis clone G3 was used throughout this study and was routinely grown in modified Diamond's medium (MDM) at $37^{\circ} \mathrm{C}$ as described previously by Lockwood et al. (1984).

Experimental incubations of $\boldsymbol{T}$. vaginalis. All experimental incubations were carried out in triplicate and on multiple occasions using the following media.

Simple media. Several media were used: (1) HEPES, $0 \cdot 1 \mathrm{M}$ HEPES, pH 7.5; (2) HEPES + maltose, HEPES supplemented with maltose $(0.5 \%, w / v, 13.9 \mathrm{mM})$; (3) HEPES + AAM, HEPES supplemented to $12 \%(\mathrm{v} / \mathrm{v})$ with a mixture of amino acids [AAM, comprising a $2: 1, \mathrm{v} / \mathrm{v}$, mixture of MEM amino acids solution without glutamine $(50 \times)$ and MEM non-essential amino acids solution $(100 \times)$; this contained 19 amino acids and was adjusted to $\mathrm{pH} 7.0$ before use]; (4) HEPES+ $\mathrm{AAM}+$ maltose, HEPES + AAM supplemented with maltose to $0.5 \%(\mathrm{w} / \mathrm{v})$; (5) HEPES + single amino acids, HEPES supplemented with L-amino acids individually at $10 \mathrm{mM}(0 \cdot 1 \mathrm{M}$ stock solutions of individual amino acids were made up in $0.1 \mathrm{M}$ HEPES; the final $\mathrm{pH}$ was adjusted to 7.5). Parasites from late exponential phase cultures in MDM were harvested by centrifugation $(1500 \mathrm{~g}, 10 \mathrm{~min})$, washed three times with $0.1 \mathrm{M}$ HEPES, $\mathrm{pH} 7 \cdot 5$, and then added to the simple media to a density of $3.0-3.5 \times 10^{6} \mathrm{ml}^{-1}$. All incubations were performed using $8 \mathrm{ml}$ medium in closed $110 \times 16 \mathrm{~mm}$ screw-cap tubes at $37^{\circ} \mathrm{C}$ 
with air as the gas phase. The densities of motile cells (flagella motility was taken as the criterion of cell viability) were determined hourly using a haemocytometer.

Complex media. The following media were used: (1) $\operatorname{MDM}(+)$, standard MDM which contains $0.5 \%$ (w/v) maltose; (2) $\operatorname{MDM}(-), \operatorname{MDM}$ without maltose; (3) $\operatorname{MDM}(-)+$ single amino acids, $\operatorname{MDM}(-)$ supplemented with individual amino acids at $10 \mathrm{mM}$ [0.1 $\mathrm{M}$ stock solutions of individual amino acids were made up in $\mathrm{MDM}(-)$ and the final $\mathrm{pH}$ was adjusted to $7 \cdot 0]$. The parasites were harvested as for the experiments with simple media, inoculated to a density of $10^{4} \mathrm{ml}^{-1}$ in $8 \mathrm{ml}$ complex medium and incubated at $37^{\circ} \mathrm{C}$ in closed tubes with air as the gas phase. Cells densities were determined daily for $4 \mathrm{~d}$.

\section{Analysis of amino acid concentrations in media using HPLC}

Sample preparation. Aliquots $(0.2 \mathrm{ml})$ were taken hourly from the incubations in simple media and daily for the growth experiments. The time 0 aliquots were taken immediately after inoculation of the parasites. The cells were removed from the aliquots by centrifugation $(10000 \mathrm{~g}, 10 \mathrm{~min})$ and the resultant cell-free samples deproteinized by addition of 4 vols cold methanol, incubation for $10 \mathrm{~min}$ at $4{ }^{\circ} \mathrm{C}$, and centrifugation as previously. The supernatants were used immediately or stored at $-20^{\circ} \mathrm{C}$

Analysis using HPLC. Samples containing amino acids were mixed with 4 vols 0 -phthaldialdehyde-thiol (OPT) reagent, prepared as described by Joseph \& Marsden (1986), immediately before being subjected to HPLC analysis. A Rainin Dyn Microsorb C18 $4.6 \times 150 \mathrm{~mm}$ column was used. Solvent A was $0.02 \mathrm{M}$ sodium acetate/methanol/tetrahydrofuran ( $85: 11: 4$, by vol.) and solvent $B$ was acetonitrile. The flow rate was $1.3 \mathrm{ml} \mathrm{min}$ and elution was effected using $0-18 \%$ solvent $B$ over the first $13 \mathrm{~min}, 18-30 \%$ solvent $\mathrm{B}$ over $11 \mathrm{~min}$ and $30-0 \%$ solvent $\mathrm{B}$ over $2 \mathrm{~min}$, with $4 \mathrm{~min}$ subsequent re-equilibration with solvent A. Detection was with a Gilson model 121 fluorometer. All mobile phases were continuously degassed with helium. Amino acids were identified according to their retention times and quantified by use of standard calibration curves. Fifteen peaks were detected when complex medium was analysed. Twelve corresponded to individual amino acids, but three peaks each contained two amino acids (histidine/glutamine, tryptophan/ phenylalanine and valine/methionine) and so the concentrations of these individual amino acids could not be determined. Neither cysteine nor proline react with OPT and so were not detected. AAM does not contain glutamine, which aided data interpretation for the experiments using simple media. The final peak eluted (lysine) proved inconsistent and so was excluded from the study. All samples were analysed by HPLC at least three times except where indicated.

Chemicals. MEM amino acids solutions were from Gibco, tetrahydrofuran and methanol were from Scotlab and acetonitrile was from Applied Biosystems. All other chemicals were from Sigma.

\section{RESULTS}

\section{Simple media}

The number of motile parasites gradually declined during the incubation in HEPES buffer alone, such that only approximately $50 \%$ remained motile by $4 \mathrm{~h}$ (Fig. 1). Addition of the amino acid mixture at $12 \%(\mathrm{v} / \mathrm{v})$ had a considerable beneficial effect, with significant cell death occurring only subsequent to $3 \mathrm{~h}$. Parasites survived less

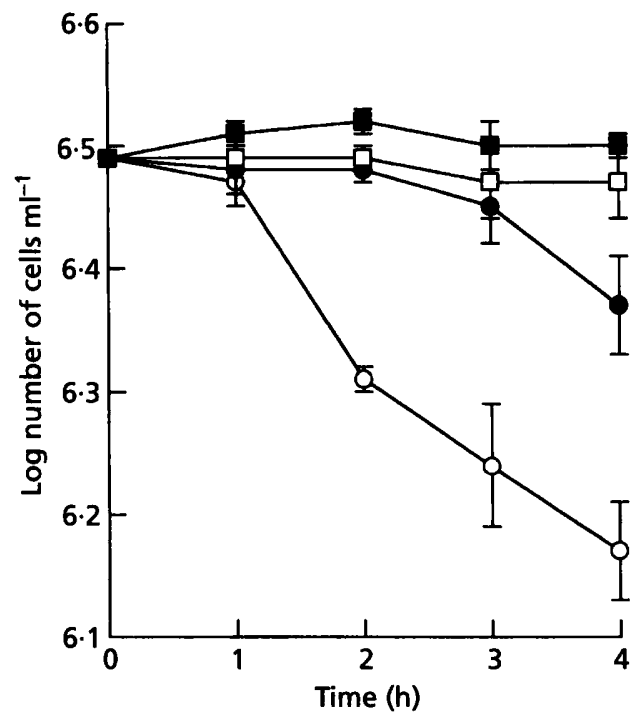

Fig. 1. Effects of supplementing simple medium with amino acids and maltose on the number of motile $T$. vaginalis. Cell densities are means $\pm S D ; n=3$. O. HEPES; O, HEPES + AAM; $\square$, HEPES + maltose; $\square$, HEPES + AAM + maltose.

well in media containing the AAM at $3 \%$ (data not shown). When maltose was added, all parasites survived for $4 \mathrm{~h}$. Some individual amino acids, notably arginine, threonine and leucine, also aided parasite survival such that the numbers remaining after $3 \mathrm{~h}$ in buffer supplemented individually with these amino acids were (mean $\log$ densities, $n=3$ ) $6.42 \pm 0.03,6.38 \pm 0.04$ and $6.37 \pm 0.02$, respectively, whereas only $6.26 \pm 0.03$ remained in buffer alone (log initial number of cells $\left.\mathrm{ml}^{-1}: 6 \cdot 54\right)$.

The changes in concentrations of amino acids during incubations of $T$. vaginalis over $3 \mathrm{~h}$ in the simple media are given in Table 1. The amino acids are listed in the order in which they were eluted from the column, aspartate being the first. The concentrations of most amino acids decreased over $3 \mathrm{~h}$ in both the media, but those in the maltose-free medium decreased more. This was also true for the co-eluted valine/methionine and tryptophan/ phenylalanine peaks. Arginine, threonine and leucine were the amino acids consumed in greatest amounts by $T$. vaginalis in the maltose-free medium. The concentration of alanine increased in both media, but especially when maltose was present. The concentration of glycine was minimally decreased in HEPES + AAM whereas a very small amount was released into the medium containing maltose.

\section{Complex media}

Of the complex media used in this study, T. vaginalis grew best in $\operatorname{MDM}(+)$ (Fig. 2). The maximum cell density reached in $\operatorname{MDM}(+)$ was considerably greater than that in $\operatorname{MDM}(-)$. T. vaginalis lived for only $3 \mathrm{~d}$ in $\operatorname{MDM}(+)$, probably because the $\mathrm{pH}$ decreased very quickly (Fig. 2), 
Table 1. Changes in amino acid concentrations ( $\mathrm{mM}$ ) during the suspension of $T$. vaginalis in simple media

Values shown are means $\pm \mathrm{SD} ; n=3$.

\begin{tabular}{|c|c|c|c|}
\hline \multirow[t]{2}{*}{ Amino acid } & \multirow{2}{*}{$\begin{array}{c}\text { Initial } \\
\text { concentrations }\end{array}$} & \multicolumn{2}{|c|}{ Changes over $3 \mathrm{~h}$} \\
\hline & & HEPES + AAM & $\begin{array}{c}\text { HEPES + AAM }+ \\
\text { maltose }\end{array}$ \\
\hline Aspartic acid & $0.364 \pm 0.017$ & $-0.083 \pm 0.020$ & $-0.017 \pm 0.011$ \\
\hline Glutamic acid & $0.510 \pm 0.021$ & $-0.136 \pm 0.046$ & $-0.014 \pm 0.012$ \\
\hline Asparagine & $0.462 \pm 0.018$ & $-0.167 \pm 0.105$ & $-0.019 \pm 0.018$ \\
\hline Serine & $0.371 \pm 0.008$ & $-0 \cdot 110 \pm 0 \cdot 041$ & $-0.016 \pm 0.019$ \\
\hline Histidine & $0.498 \pm 0 \cdot 018$ & $-0.138 \pm 0.045$ & $-0.017 \pm 0.019$ \\
\hline Glycine & $0 \cdot 236 \pm 0.001$ & $-0.029 \pm 0.008$ & $+0.009 \pm 0.007$ \\
\hline Threonine & $1.890 \pm 0.075$ & $-0.608 \pm 0.111$ & $-0.194 \pm 0.114$ \\
\hline Alanine & $0.471 \pm 0.007$ & $+0.010 \pm 0.007$ & $+0.130 \pm 0.070$ \\
\hline Arginine & $2.062 \pm 0.063$ & $-0.658 \pm 0.154$ & $-0.201 \pm 0.077$ \\
\hline Tyrosine & $1 \cdot 023 \pm 0 \cdot 040$ & $-0.275 \pm 0.089$ & $-0.069 \pm 0.070$ \\
\hline Isoleucine & $1 \cdot 214 \pm 0 \cdot 040$ & $-0.326 \pm 0.071$ & $-0 \cdot 109 \pm 0.029$ \\
\hline Leucine & $1.567 \pm 0.050$ & $-0.409 \pm 0.095$ & $-0.105 \pm 0.032$ \\
\hline
\end{tabular}

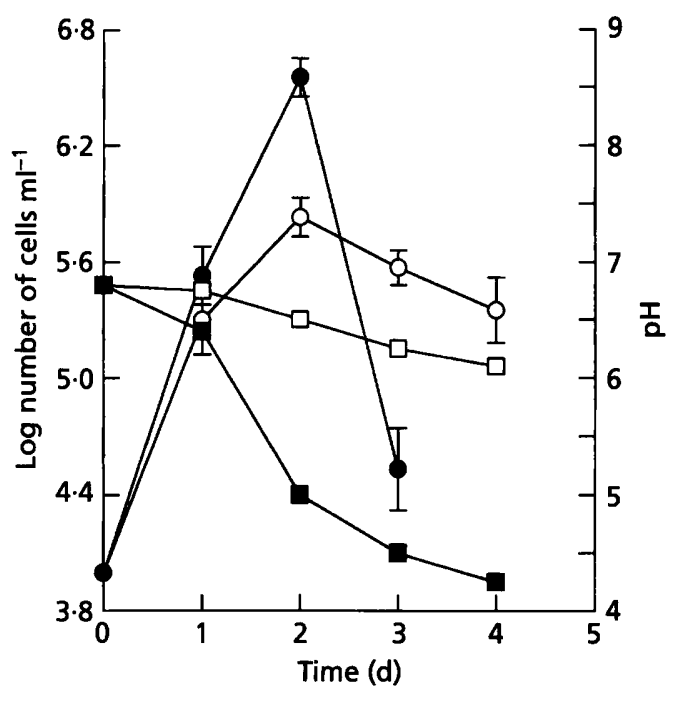

Fig. 2. Comparison of the growth of $T$. vaginalis in $M D M(-)$ and $M D M(+)$. Cell densities are means $\pm S D ; n=3$. All cells in $M D M(+)$ died by day 4 of the culture. $O$, Cell density in $\operatorname{MDM}(-) ; \square, \mathrm{pH}$ of $\operatorname{MDM}(-)$; 0 , cell density in $\operatorname{MDM}(+)$; a. $\mathrm{pH}$ of $\mathrm{MDM}(+)$.

whereas some cells remained viable for 6-7 d in $\operatorname{MDM}(-)$ (data not shown). The amino acid concentrations were analysed only for the first $3 \mathrm{~d}$, during which most cells were living and there was insignificant cell lysis.

The changes in concentrations of amino acids in $\operatorname{MDM}(-)$ and $\operatorname{MDM}(+)$ over $3 \mathrm{~d}$ of $T$. vaginalis culture are shown in Table 2. Most amino acids decreased in concentration over the $3 \mathrm{~d}$, with the decreases in many cases being somewhat greater in $\operatorname{MDM}(-)$ than in $\operatorname{MDM}(+)$. The unresolved valine/methionine and tryptophan/phenylalanine peaks also decreased, the latter very considerably. Leucine was consumed in particularly large amounts, with concentrations of arginine, glutamic acid, threonine and serine also decreasing greatly. Alanine and glycine increased in concentration in both media, but the increases were more than twofold greater in $\operatorname{MDM}(+)$.

Twenty L-amino acids were individually added to $\operatorname{MDM}(-)$ and their effects on the growth of $T$. vaginalis were studied. It was found that six amino acids (arginine, threonine, leucine, isoleucine, glutamic acid and phenylalanine) had beneficial effects on the growth of $T$. vaginalis in the absence of maltose (Fig. 3). The cell densities of $T$. vaginalis cultured in the $\operatorname{MDM}(-)$ supplemented with these amino acids were markedly higher on days 2 and 3 than in $\operatorname{MDM}(-)$ alone, and greater amounts of the amino acids were consumed. Methionine, glutamine and homoserine, when added in the same way, also potentiated growth, but to a smaller extent (data not shown).

\section{DISCUSSION}

The results have shown that $T$. vaginalis grows or survives much better in the media containing maltose than in the maltose-free media (Figs 1 and 2). These results support the view that the major energy substrates for this parasite are carbohydrates (Müller, 1988, 1991; Coombs \& Müller, 1995). Amino acids, however, also aided parasite survival (Fig. 1) and indeed growth (Fig. 3) in the absence of added maltose, and it was also found that $T$. vaginalis consumes larger amounts of amino acids in the absence of maltose than in its presence (Tables 1 and 2). These data are consistent with the idea that amino acids may be used as energy substrates by $T$. vaginalis, but they also suggest that such use is relatively unimportant when carbohydrates are 
Table 2. Changes in amino acid concentrations (mM) during the growth of $T$. vaginalis in complex media

Values shown are means $\pm \mathrm{sD} ; n=3$.

\begin{tabular}{|c|c|c|c|}
\hline \multirow[t]{2}{*}{ Amino acid } & \multirow{2}{*}{$\begin{array}{c}\text { Initial } \\
\text { concentrations }\end{array}$} & \multicolumn{2}{|c|}{ Changes over $3 \mathrm{~d}$} \\
\hline & & $\operatorname{MDM}(-)$ & $\operatorname{MDM}(+)$ \\
\hline Aspartic acid & $1.92 \pm 0.09$ & $-0.25 \pm 0.21$ & $-0.15 \pm 0.06$ \\
\hline Glutamic acid & $7 \cdot 31 \pm 0.52$ & $-0.53 \pm 0.38$ & $-0.43 \pm 0.33$ \\
\hline Asparagine & $1.87 \pm 0.05$ & $-0 \cdot 16 \pm 0 \cdot 15$ & $-0 \cdot 17 \pm 0 \cdot 12$ \\
\hline Serine & $2 \cdot 83 \pm 0 \cdot 20$ & $-0 \cdot 39 \pm 0 \cdot 15$ & $-0.37 \pm 0.22$ \\
\hline Glycine & $1.70 \pm 0.07$ & $+0 \cdot 30 \pm 0 \cdot 18$ & $+0.71 \pm 0.14$ \\
\hline Threonine & $3 \cdot 11 \pm 0 \cdot 11$ & $-0.46 \pm 0.34$ & $-0.29 \pm 0.07$ \\
\hline Alanine & $6 \cdot 50 \pm 0 \cdot 40$ & $+1.37 \pm 0.53$ & $+3 \cdot 26 \pm 0 \cdot 16$ \\
\hline Arginine & $2 \cdot 87 \pm 0 \cdot 24$ & $-0.56 \pm 0.03$ & $-0.37 \pm 0.15$ \\
\hline Tyrosine & $1.58 \pm 0.02$ & $-0.11 \pm 0.03$ & $-0.03 \pm 0.02$ \\
\hline Isoleucine & $2 \cdot 95 \pm 0.02$ & $-0.24 \pm 0.28$ & $-0.08 \pm 0.11$ \\
\hline Leucine & $10 \cdot 46 \pm 0 \cdot 71$ & $-1 \cdot 24 \pm 0 \cdot 42$ & $-0.95 \pm 0.11$ \\
\hline
\end{tabular}
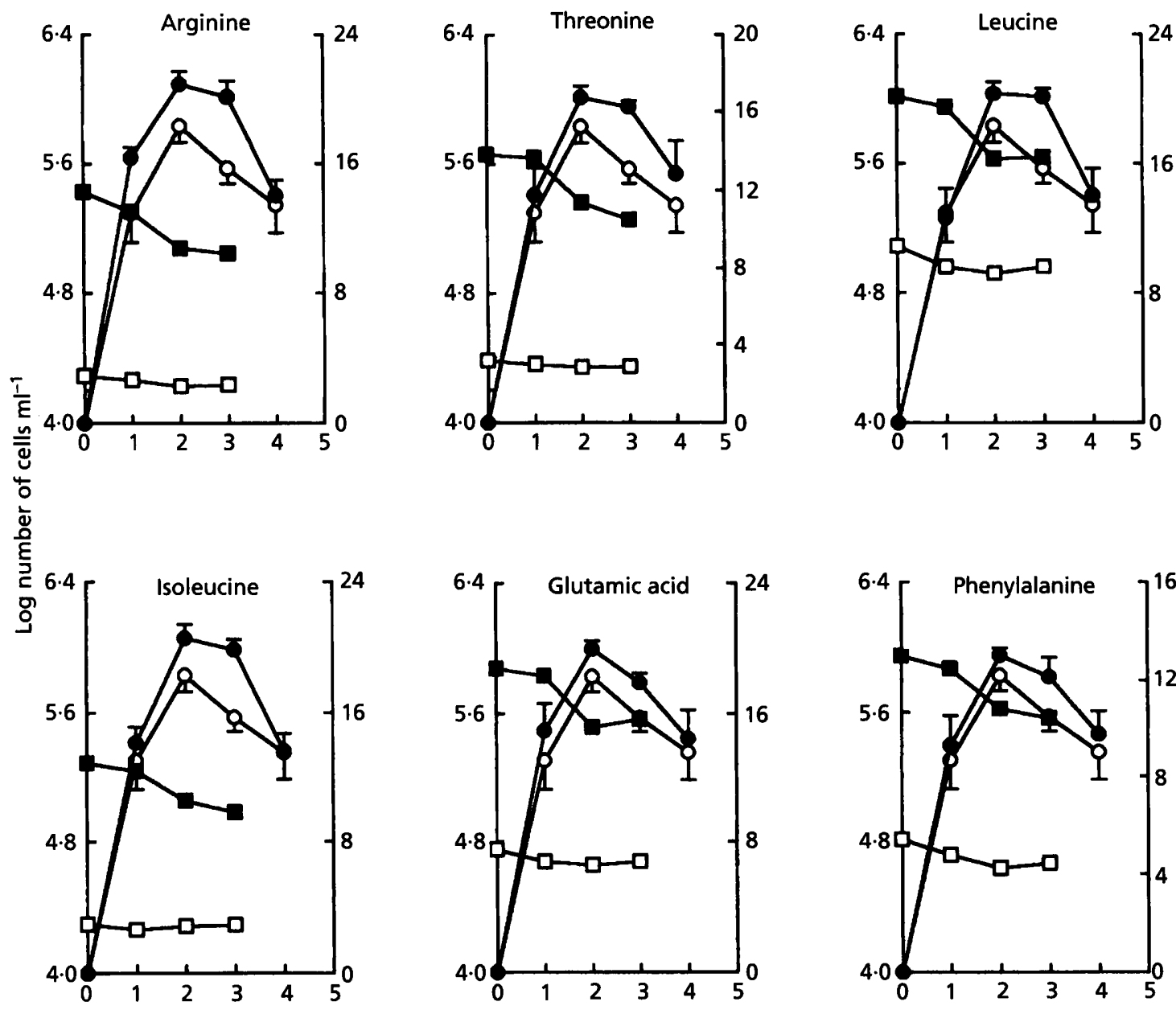

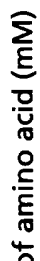
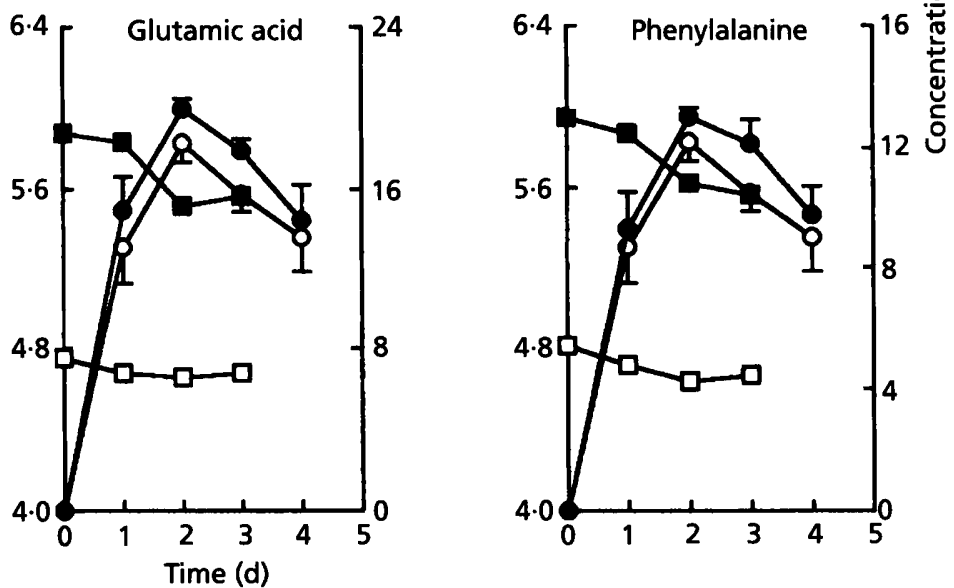

Fig. 3. Effects of supplementing $M D M(-)$ with individual amino acids on the growth of $T$. vaginalis. Cell densities are means $\pm S D ; n=3$. 0 . Cell density in $\mathrm{MDM}(-)$; 0 , cell density in $\mathrm{MDM}(-)+$ amino acid $(10 \mathrm{mM}) ; \square$, concentration of amino acid in $M D M(-) ; \square$, concentration of amino acid in $M D M(-)+$ amino acid $(10 \mathrm{mM})$. 
present. Presumably amino acids are mainly taken up for other purposes, such as protein synthesis, when maltose is abundantly available.

It is interesting that there was found to be little difference between the survival of the parasite during the first hour of suspension in the various simple media. This is perhaps a reflection of the residual energy substrates, such as glycogen, in the cells. The data obtained using the complex media have to be interpreted with caution. Though MDM(-) was devoid of added maltose, it still contained the small amounts of carbohydrates present in the other constituents of the medium. It is likely that these carbohydrates would have been completely consumed by $T$. vaginalis during the initial phase of the culture, but their presence may well explain why there were found to be no large differences between the first day of growth of $T$. vaginalis in $\mathrm{MDM}(-), \operatorname{MDM}(+)$, and $\mathrm{MDM}(-)$ supplemented with single amino acids. MDM also contains proteins which could be used by the parasite as a source of amino acids. This may account for the relatively low consumption of free amino acids from MDM compared with the consumption from the simple media (compare Tables 1 and 2). Nevertheless, the data from the experiments using MDM show that amino acids were consumed in significant quantities.

Previous studies have shown that $T$. vaginalis rapidly consumes arginine from the environment and catabolizes it through an energy-yielding pathway (Linstead \& Cranshaw, 1983). It has also been reported that methionine is rapidly consumed and catabolized by the parasite in vitro (Thong et al., 1987; Lockwood \& Coombs, 1989, 1991a). Enzymes that could utilize leucine and threonine, leucine aminotransferase and threonine dehydratase, respectively, have also been detected at high activity in the parasite (Lowe \& Rowe, 1986; Lockwood \& Coombs, 1991b). The current study has now provided evidence supporting the suggestion that arginine, threonine and leucine are used as energy substrates in the absence of carbohydrates. The results show, however, that several other amino acids are also consumed by $T$. vaginalis and are beneficial to its growth or survival in the maitose-free media and so suggest that the parasite may be able to use several amino acids as energy substrates.

The major end-products known to be excreted by growing $T$. vaginalis are glycerol, lactate, acetate, $\mathrm{CO}_{2}$ and $\mathrm{H}_{2}$ (Müller, 1988, 1991; Coombs \& Müller, 1995), with some alanine also being produced from glucose (Chapman et al., 1985). The results of this study show that glycine should be added to this list of products. It is interesting that alanine and glycine were both excreted by $T$. vaginalis in greater amounts when maltose was present. This may simply reflect the higher rate of metabolism supported by maltose, but could also be due to different pathways operating when maltose is absent. It has been shown that Giardia lamblia, another anaerobic, parasitic protist, also produces alanine as a major end-product of carbohydrate metabolism (Edwards et al., 1989), but only under anaerobic conditions (Paget et al., 1990). Oxygen was not removed in setting up the cultures in the current study, although it is likely that the oxygen initially present in the complex medium would have been removed relatively quickly by the growing parasites. Thus it is possible that the relatively small net production of alanine by $T$. vaginalis suspended for short periods in the simple media, compared with the production when growing in complex media, could reflect, in part, the different concentrations of oxygen that were present during the incubations. Glycine is also excreted by $G$. lamblia (Edwards et al., 1989) and we have recently found that Entamoeba histolytica and $E$. invadens release significant amounts of both alanine and glycine when growing axenically in culture, and that the production is greater when glucose is present (X. Zuo and others, unpublished).

T. vaginalis, G. lamblia and the two species of Entamoeba are all lumen-dwelling parasitic protists and although they are not phylogenetically very close they do share a number of features, such as a fermentative metabolism and the apparent lack of mitochondria, which are considered to be adaptations for anaerobiosis (Müller, 1988; Coombs \& Müller, 1995). It has been found that Giardia and the Entamoeba species also consume arginine and other amino acids (Schofield et al., 1990; Schofield \& Edwards, 1991, 1994; North \& Lockwood, 1995; X. Zuo and others, unpublished) and so it now appears that an ability to catabolize some amino acids for energy production, and release alanine and glycine as end-products of this breakdown, may be adaptations for anaerobiosis that are common features of this group of anaerobic, parasitic protists.

This study has provided the first quantitative data on the consumption of a range of amino acids by $T$. vaginalis. These baseline data pave the way for more detailed investigations. The intention now is to determine how amino acid consumption varies with environmental conditions (for example, how the rate of uptake depends upon the availability of individual amino acids), the pathways by which the individual amino acids are broken down, the subcellular location of these pathways, and how the catabolism of amino acids is regulated.

\section{ACKNOWLEDGEMENTS}

X.Z. was supported by the British Council and B.C.L. was a Royal Society University Research Fellow.

\section{REFERENCES}

Chapman, A., Linstead, D. J., Lloyd, D. \& Williams, J. (1985). ${ }^{13} \mathrm{C}-$ NMR reveals glycerol as an unexpected major metabolite of the protozoan parasite Trichomonas vaginalis. FEBS Lett 191, 287-292.

Coombs, G. H. \& Muller, M. (1995). Energy metabolism of anaerobic parasitic protists. In Biochemistry and Molecular Biology of Parasitic Organisms. Edited by J. J. Marr \& M. Müller. London: Academic Press (in press).

Edwards, M. R., Gilroy, F. V., Jimenez, M. B. \& O'Sullivan, W. J. (1989). Alanine is a major product of metabolism by Giardia lamblia: a proton magnetic resonance study. Mol Biochem Parasitol 37, 19-26.

Honigberg, B. M. (editor) (1989). Trichomonads Parasitic in Humans. New York: Springer-Verlag. 
Joseph, M. H. \& Marsden, C. A. (1986). Amino acids and small peptides. In HPLC of Small Molecules, a Practical Approach, pp. 13-28. Edited by D. Rickwood \& B. D. Hames. Oxford: IRL Press.

Linstead, D. \& Cranshaw, M. (1983). The pathway of arginine catabolism in the parasitic flagellate Trichomonas vaginalis. Mol Biochem Parasitol 8, 241-252.

Lockwood, B. C. \& Coombs, G. H. (1989). The catabolism of amino acids by Trichomonas vaginalis. In Biochemistry and Molecular Biology of 'Anaerobic' Protozoa, pp. 93-111. Edited by D. Lloyd, G. H. Coombs \& T. A. Paget. Chur: Harwood.

Lockwood, B. C. \& Coombs, G. H. (1991a). Purification and characterisation of methionine $\gamma$-lyase from Trichomonas vaginalis. Biocbem J 279, 675-682.

Lockwood, B. C. \& Coombs, G. H. (1991b). Amino acids catabolism in anaerobic protists. In Biochemical Protozoology, pp. 113-122. Edited by G. H. Coombs \& M. J. North. London: Taylor \& Francis.

Lockwood, B. C., North, M. J. \& Coombs, G. H. (1984). Trichomonas vaginalis, Tritrichomonas foetus and Trichomitus batrachorum: comparative proteolytic activity. Exp Parasitol 58, 245-253.

Lowe, P. N. \& Rowe, A. F. (1986). Aminotransferase activities in Trichomonas vaginalis. Mol Biochem Parasitol 21, 65-74.

Muller, M. (1988). Energy metabolism of protozoa without mitochondria. Annu Rev Microbiol 42, 465-488.
Muller, M. (1991). Energy metabolism of anaerobic parasitic protists. In Biochemical Protozoology, pp. 80-91. Edited by G. H. Coombs \& M. J. North. London: Taylor \& Francis.

North, M. J. \& Lockwood, B. C. (1995). Amino acid and protein metabolism. In Biochemistry and Molecular Biology of Parasitic Organisms. Edited by J. J. Marr \& M. Müller. London: Academic Press (in press).

Paget, T. A., Raynor, M. H., Shipp, D. W. E. \& Lloyd, D. (1990). Giardia lamblia produces alanine anaerobically but not in the presence of oxygen. Mol Biochem Parasitol 41, 65-72.

Schofield, P. J. \& Edwards, M. R. (1991). Energy metabolism in Giardia intestinalis. In Biocbemical Protozoology, pp. 102-112. Edited by G. H. Coombs \& M. J. North. London: Taylor \& Francis.

Schofield, P. J. \& Edwards, M. R. (1994). Biochemistry - is Giardia opportunistic in its use of substrates? In Giardia: from Molecules to Disease, pp. 171-183. Edited by R. C. A. Thompson, J. A. Reynoldson \& A. J. Lymbery. Wallingford: CAB International.

Schofield, P. J., Costello, M., Edwards, M. R. \& O'Sullivan, W. J. (1990). The arginine dihydrolase pathway is present in Giardia intestinalis. Int J Parasitol 20, 697-699.

Thong, K.-W., Coombs, G. H. \& Sanderson, B. E. (1987). LMethionine catabolism in trichomonads. Mol Biochem Parasitol 23, 223-231.

Received 20 May 1995; accepted 22 June 1995. 